\title{
Intervening upregulated SLC7A5 could mitigate inflammatory mediator by mTOR- P70S6K signal in rheumatoid arthritis synoviocytes
}

Jing $X u^{1,2+}$, Congshan Jiang ${ }^{1,2+}$, Yongsong Cai ${ }^{3}$, Yuanxu Guo ${ }^{1,2}$, Xipeng Wang ${ }^{1,2}$, Jiaxiang Zhang ${ }^{1,2}$, Jiawen Xu ${ }^{3}$,

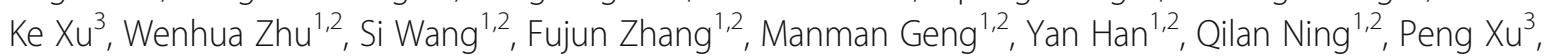
Liesu Meng ${ }^{1,2}$ and Shemin $L^{1,2^{*}}$ (D)

\begin{abstract}
Objective: The disruption of metabolic events and changes to nutrient and oxygen availability due to sustained inflammation in RA increases the demand of bioenergetic and biosynthetic processes within the damaged tissue. The current study aimed to understand the molecular mechanisms of SLC7A5 (amino acid transporter) in synoviocytes of RA patients.

Methods: Synovial tissues were obtained from OA and RA patients. Fibroblast-like synoviocytes (FLS) were isolated, and SLC7A5 expression was examined by using RT-qPCR, immunofluorescence, and Western blotting. RNAi and antibody blocking treatments were used to knockdown SLC7A5 expression or to block its transporter activities. mTOR activity assay and MMP expression levels were monitored in RA FLS under amino acid deprivation or nutrient-rich conditions.
\end{abstract}

Results: RA FLS displayed significantly upregulated expression of SLC7A5 compared to OA FLS. Cytokine IL-1 $\beta$ was found to play a crucial role in upregulating SLC7A5 expression via the NF-KB pathway. Intervening SLC7A5 expression with RNAi or blocking its function by monoclonal antibody ameliorated MMP3 and MMP13 protein expression. Conversely, upregulation of SLC7A5 or tryptophan supplementation enhanced mTOR-P70S6K signals which promoted the protein translation of MMP3 and MMP13 in RA FLS.

Conclusion: Activated NF-KB pathway upregulates SLC7A5, which enhances the mTOR-P70S6K activity and MMP3 and MMP13 expression in RA FLS.

Keywords: SLC7A5, MMPs, mTOR-P70S6K, Fibroblast-like synoviocytes, Rheumatoid arthritis

\footnotetext{
* Correspondence: lushemin@xjtu.edu.cn

${ }^{\dagger}$ Jing $\mathrm{Xu}$ and Congshan Jiang are co-first authors.

'Department of Biochemistry and Molecular Biology, School of Basic Medical

Sciences, Xi'an Jiaotong University Health Science Center, Xi'an 710061,

Shaanxi, People's Republic of China

${ }^{2}$ Key Laboratory of Environment and Genes Related to Diseases (Xi'an

Jiaotong University), Ministry of Education, Xi'an 710061, Shaanxi, People's

Republic of China

Full list of author information is available at the end of the article
}

(c) The Author(s). 2020 Open Access This article is licensed under a Creative Commons Attribution 4.0 International License, which permits use, sharing, adaptation, distribution and reproduction in any medium or format, as long as you give appropriate credit to the original author(s) and the source, provide a link to the Creative Commons licence, and indicate if changes were made. The images or other third party material in this article are included in the article's Creative Commons licence, unless indicated otherwise in a credit line to the material. If material is not included in the article's Creative Commons licence and your intended use is not permitted by statutory regulation or exceeds the permitted use, you will need to obtain permission directly from the copyright holder. To view a copy of this licence, visit http://creativecommons.org/licenses/by/4.0/ The Creative Commons Public Domain Dedication waiver (http://creativecommons.org/publicdomain/zero/1.0/) applies to the data made available in this article, unless otherwise stated in a credit line to the data. 


\section{Background}

Rheumatoid arthritis (RA) is a chronic autoimmune disease with a global prevalence of $0.24 \%$ [1], characterized by synovial hyperplasia and progressive destruction of mainly the small joints. Many cell types, including $\mathrm{T}$ cells, B cells, macrophages, and fibroblast-like synoviocytes (FLSs), participate in the complex mechanism of RA pathogenesis. FLSs in the lining of the synovium play a major role and express high levels of inflammatory cytokines that perpetuate inflammation and proteases that degrade the cartilage [2]. Furthermore, RA FLSs are described to present a tumor-like phenotype [3], with increased invasiveness into the extracellular matrix (ECM), which further exacerbates synovial hyperplasia and joint damage $[4,5]$. Meanwhile, these quickly proliferated FLS demand high energy, which is well associated with highlevel transportation and consumption of glucose and amino acids.

Solute carrier family 7 member 5 (SLC7A5), alias Ltype amino acid transporter (LAT1) [6], is a sodiumindependent high-affinity amino acid transporter. SLC7A5 together with SLC3A2 mediates cellular uptake of the large neutral amino acids such as phenylalanine, tyrosine, leucine, and tryptophan [7]. The SLC7A5 is mainly distributed in the placenta, testis, bone marrow, and brain, whereas $S L C 3 A 2$ is expressed ubiquitously in all tissues [8]. Global knockout of Slc7a5 resulted in an embryonic lethal phenotype in mice, and it may be partly due to a deleterious effect upon Slc7a5 transport function during post-implantation embryonic development $[9,10]$. The conditional knockout of Slc7a5 showed that Slc7a5 worked as a checkpoint in T cell activation via the mTORC1 complex [11]. Meanwhile, the hypoxia-inducible factor $2 \alpha$ binds to the SLC7A5 proximal promoter and drives its transcription in the WT8 cell line [12]. In the inflamed RA joints, the hypoxic condition becomes gradually severe due to increased metabolic demand of the active cells and due to inadequate oxygen delivery through poor perfusion of the inflamed joint [13]. Recently, an mRNA expression profiling study has documented the elevated levels of SLC7A5 in RA synovial tissue [14].

Different studies have indicated the potential role of SLC7A5 in RA pathogenesis; however, much is not known about its actual function in the inflamed FLS. This study was proposed to explore the potential role of SLC7A5 and understand the underlying molecular mechanism in FLS of RA patients.

\section{Methods}

\section{Patients' samples}

Synovial tissues and FLS were derived from patients with RA and OA who underwent surgical knee joint replacement (Department of Joint Surgery, Honghui Hospital,
Xi'an Jiaotong University, China). All the patients' data are summarized in Table 1. All participants gave their written informed consent prior to inclusion in the study. The study was approved by the Medical Ethics Committee of Xi'an Jiaotong University (No. 2016-261 and No.2017-666).

\section{Histology and immunofluorescence}

For routine histopathological analysis, paraffinembedded synovial tissue sections from RA and OA patients were deparaffinized and stained with hematoxylin and eosin (H\&E). For immunofluorescence staining, 6$\mu \mathrm{m}$-thick tissue sections were incubated overnight at $4{ }^{\circ} \mathrm{C}$ with the following primary antibodies diluted in PBS: mouse monoclonal antibody against SLC7A5 (1: 100, Santa Cruz, sc-374232) and rabbit polyclonal antibody to vimentin (1:100, Bioss, bs-23064R). Next morning, the samples were washed three times in PBS and incubated for $45 \mathrm{~min}$ at room temperature with secondary antibodies, i.e., FITC AffiniPure goat anti-mouse IgG $(\mathrm{H}+\mathrm{L})$ (1:400, Earthox, E031210-01) and Cy3 AffiniPure goat anti-rabbit IgG (H+L) (1:400, Earthox, E031620-01). 4',6-Diamidino-2-phenylindole (DAPI) was used to detect the nucleus $(1: 100,000$, Sigma-Aldrich, D9542). Immunofluorescent staining procedure was followed with slight modifications, as previously described [15]. The immunofluorescent images were captured with a fluorescence microscope (Olympus, Japan) and analyzed by the ImageJ software.

\section{Cytokines and inhibitor treatment}

Cells were treated with IL-1 $\beta$ (20 ng/mL), TNF- $\alpha(20 \mathrm{ng} /$ $\mathrm{mL})$, IFN- $\gamma(20 \mathrm{ng} / \mathrm{mL})$, IL-6 $(20 \mathrm{ng} / \mathrm{mL})$, and IL-17A (20 ng/mL) (Genscript, China) for $24 \mathrm{~h}$, and total protein analysis was performed using Western blotting assay.

The samples were incubated with JNK inhibitor SP600125 (10 $\mu \mathrm{M}$, Selleckchem, s1460), NF-kB inhibitor BAY $_{11-7085}(10 \mu \mathrm{M}$, Selleckchem, s7352), or P38 inhibitor SB203580 (10 $\mu \mathrm{M}$, MEC, HY10256A) for $4 \mathrm{~h}$, followed

Table 1 Patient characteristics

\begin{tabular}{lll}
\hline Clinical data & RA & OA \\
\hline Number of patients & 24 & 24 \\
Sex & & \\
$\quad$ Female & 17 & 19 \\
$\quad$ Male & 7 & 5 \\
Age & & $66.38 \pm 1.396$ \\
CRP $^{\#}(\mathrm{mg} / \mathrm{L})$ & $56.71 \pm 1.673$ & $4.25 \pm 1.608$ \\
$\mathrm{RF}^{\#}(\mathrm{IU} / \mathrm{mL})$ & $29.30 \pm 3.615$ & $6.846 \pm 0.9816$ \\
ESR $^{\#}(\mathrm{~mm} / \mathrm{h})$ & $92.57 \pm 15.07$ & $15.79 \pm 3.774$ \\
\hline
\end{tabular}

CRP C-reactive protein, $R F$ rheumatoid factor, ESR erythrocyte sedimentation rate

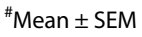


by the addition of $20 \mathrm{ng} / \mathrm{mL}$ IL- $1 \beta$ for $24 \mathrm{~h}$, to stimulate the cells. The expression at the mRNA and protein levels was determined by RT-qPCR and Western blotting, respectively.

\section{Blocking assay of SLC7A5}

SLC7A5 antibody $(20 \mu \mathrm{g} / \mathrm{mL}$, a mouse anti-SLC7A5 monoclonal antibody, $\operatorname{IgG}_{1}$, Santa Cruz, USA) was administrated to the FLS, following the procedure as detailed in our previous paper [16]. Briefly, FLSs were seeded in 12 -well plates at a density of $4 \times 10^{4} / \mathrm{mL}$ and incubated with SLC7A5 antibody or isotype-matched $\mathrm{IgG}_{1}$ (CST, \#5415, USA) for $24 \mathrm{~h}$. The cells were then treated with IL-1 $\beta$ for $18 \mathrm{~h}$ and collected to detect the mRNA and protein levels of MMP3 and MMP13.

\section{Western blotting}

Total protein lysates from synovial tissues and cells were extracted by using the RIPA solution (Beyotime, China) with a cocktail of protease and phosphatase inhibitors (Roche). The total protein concentration of each sample was determined by a BCA Protein Assay kit (Thermo Scientific, USA). Subsequently, $20 \mu \mathrm{g}$ from cell lysates was separated by $6 \%$ or $8 \%$ SDS-PAGE gels and transferred to the polyvinylidene fluoride membrane (EMD Millipore, Billerica, MA, USA). The membrane was incubated with primary antibodies at $4{ }^{\circ} \mathrm{C}$ overnight. The list of primary antibodies is depicted in supplemental Table S5. After washing, the membrane was further incubated with a horseradish peroxidase-conjugated goat anti-rabbit or goat anti-mouse IgG secondary antibody $(0.4 \mu \mathrm{g} / \mathrm{mL}$, Abcam, USA) for $2 \mathrm{~h}$ at room temperature. Signal intensity was determined by the Supersignal ${ }^{\circ}$ West Pico Kit (Thermo Scientific) using the enhanced chemiluminescence detection system (EMD Millipore). The band density was measured by the ImageJ software normalized to $\beta$-actin.

\section{RNA isolation and RT-qPCR}

Total RNA from the synovial tissues and cells was isolated using the TRI Reagent ${ }^{\text {tm }}$ solution (Thermo Scientific, USA) and reverse transcribed to CDNA using the First Strand cDNA Synthesis Kit (Thermo Scientific, USA) according to the manufacturer's instructions. RTqPCR was performed by using the iQ5 optical system software (Bio-Rad Laboratories, USA) with Fast Start Universal SYBR Green Master (ROX) (Roche, USA) for relative quantification of the target genes at mRNA level. Gene expression analyses were calculated by $2^{-\Delta \Delta C t}$ method.

\section{RNAi}

Small interfering RNAs (siRNAs) targeting SLC7A5 (si1: 5'-CATTATACAGCGGCCTCTTT-3', si2: 5'-TAGA TCCCAACTTCTCATTT-3') and the negative control
(NC, 5'-GCGACGAUCUGCCUAAGAUTT-3') were purchased from Oligobio (Beijing, China). Cells were transfected with $75 \mathrm{nmol} / \mathrm{L}$ of either SLC7A5 siRNA or NC siRNA using Lipofectamine ${ }^{\mathrm{rw}} 2000$ Transfection Reagent (Thermo Scientific, USA) according to the manufacturer's guidelines. The cells were collected for RNA or protein isolation $24-48 \mathrm{~h}$ post-transfection, where indicated to detect the treatment effects and the signal pathways.

\section{Cytokine profiling assay}

RA FLSs were seeded in 6 -well plates $\left(2 \times 10^{5}\right.$ cells $\left./ \mathrm{mL}\right)$ and incubated overnight in DMEM medium containing $5 \%$ FBS. Subsequently, the cells were transfected with siRNA, and $4 \mathrm{~h}$ later, the medium was replaced by containing $0.2 \%$ FBS and incubated for $48 \mathrm{~h}$. Supernatants were collected and centrifuged (at $2000 \mathrm{rpm}$ for $10 \mathrm{~min}$ at $4{ }^{\circ} \mathrm{C}$ ), and aliquots were stored at $-80^{\circ} \mathrm{C}$ before further analyses.

Cytokine expression in siRNA-treated RA FLS supernatants was detected by using $\mathrm{RayBio}^{\circ} \mathrm{C}$-Series human cytokine antibody array (AAH-CYT-5). Dot ELISAbased membrane coated with 80 human cytokines (listed in supplemental Table S2) was incubated with RA FLS supernatants pooled from 4 donors, transfected with si$S L C 7 A 5$ or si-NC for $48 \mathrm{~h}$. The detection and analysis of the cytokine array were performed by RayBiotech Company according to the manufacturer's instructions. Dot immunoblot signals from the membrane array were captured, and the raw intensity was calculated as shown in supplemental Table S3.

\section{Amino acid deficiency and supplement assay}

Lab self-made DMEM were followed by Dulbecco's modified Eagle's medium (DME) formulation recipe in the Sigma-Aldrich website. The single amino aciddeficient medium was prepared at the laboratory based on the Dulbecco's modified Eagle's medium (DMEM) formulation from Sigma-Aldrich lacking either phenylalanine (Phe) or tryptophan (Trp). For the amino acid supplement assay, additional $1 \mathrm{mM}$ phenylalanine (Phe), tryptophan (Trp), or kynurenine (Kyn) were added into the DMEM medium. The FLSs were cultured in a single amino acid deficiency medium initially for $8 \mathrm{~h}$ before the addition of IL-1 $\beta$ into the treatment group medium and incubated for another $16 \mathrm{~h}$. The cells were collected for mTOR activity and MMP expression analyses.

\section{Statistics}

Data were expressed as mean \pm standard error of mean and SPSS software was used for statistical analyses. Oneway ANOVA among the groups and Student's $t$ test or Mann-Whitney-Wilcoxon test between the two groups were used to determine significant differences according 
to the distribution of the data (normal distribution was validated using Shapiro-Wilk test). $p$ less than 0.05 was considered statistically significant.

\section{Results \\ SLC7A5 expression is upregulated in fibroblast-like synoviocytes from RA patients}

To investigate the involvement of SLC7A5 in RA pathogenesis, synovial tissues were collected from RA and OA patients. Histological examination revealed that the synovial tissues from RA patients were heavily proliferated and more infiltrated with inflammatory cells (blue arrow), compared with those from OA patients (Fig. 1a). The expression of $S L C 7 A 5$ at the mRNA level was significantly upregulated in synovial tissues from RA patients as compared to those from OA patients (Fig. 1b). In addition, we observed a significant positive correlation of SLC7A5 expression at the mRNA level with both RF (Fig. 1c) and CRP (Fig. 1d). Likewise, SLC7A5 expression at the protein level was also found significantly upregulated in synovial tissues from RA patients as compared to those from OA patients (Fig. 1e, Supplementary Fig. S1). Immunofluorescence staining of the synovial tissues from RA patients revealed that SLC7A5 was overexpressed and co-localized in vimentin-positive cells (FLS) (Fig. 1f, g).

\section{The upregulation of SLC7A5is mediated by IL-1 $\beta$ via the NF-KB pathway}

To scrutinize which molecule is responsible for the upregulation of SLC7A5 in FLS, we focused on proinflammatory cytokines, the chief sponsors of inflammation in RA. Interestingly, we found that both IL-1 $\beta$ and IL- 6 could significantly upregulate SLC7A5 expression at the protein level (Fig. 2a). We used IL-1 $\beta$ to activate both the JNK and NF$\kappa B$ signaling pathways (Fig. $2 b$ ) in FLS, either by phosphorylating JNK or promoting I $\kappa B$ degradation. To know the underlying pathway involved in upregulating SLC7A5 expression, the cells were treated with SP600125 (JNK signaling inhibitor) or Bay ${ }_{11-7085}$ (NF- $\mathrm{kB}$ signaling inhibitor) and stimulated by IL-1 $\beta$. The data revealed that the activated NF-KB signaling played a fundamental role in SLC7A5 upregulation (Fig. 2c, d, Supplementary Fig. S3). We also used SB203580 (P38 pathway inhibitor) in IL-1 $\beta$-stimulated cells; however, the data showed no involvement of P38 signaling in SLC7A5 upregulation (Fig. 2e).

\section{Upregulated SLC7A5 enhances MMP3 and MMP13 protein expression in FLS}

To figure out the function of SLC7A5 as an amino acid transporter in activated FLS, the SLC7A5 monoclonal antibody was used as a blocker. RT-qPCR results showed that there was no change in the MMP3 and MMP13 expression at the mRNA level (Fig. 3a). However, the protein levels of MMP3 and MMP13 were decreased by the SLC7A5 blocker antibody (Fig. 3b, Supplementary Fig. S4A), indicating that the suppression of MMP3 and MMP13 happened only at the protein level.

Two sequences of small interfering RNAs specific to SLC7A5 were synthesized and optimized. siRNA No.2 was found to downregulate the expression of SLC7A5 significantly in FLS $48 \mathrm{~h}$ post-transfection both at the protein (Fig. 3c) and mRNA (Fig. 3d) levels. Although the siRNA downregulated the SLC7A5 expression at the mRNA level successfully, there was no change in the mRNA levels of MMP3 and MMP13 (Fig. 3e-g). However, the protein levels of MMP3 and MMP13 were found downregulated by the SLC7A5 siRNA (Fig. 3h, Supplementary Fig. S4B). These results uncovered the involvement of SLC7A5 in regulating MMP3 and MMP13 proteins in RA FLS.

A total of 80 human cytokines were detected (Fig. 4a) in conditioned media of RA FLSs transfected with SLC7A5 siRNA for $48 \mathrm{~h}$ (Fig. 3c). Semi-quantitative data showed that the fold change increase in the expression of IL-10, PARC, PLGF, TGF $\beta_{2}, \mathrm{TGF}_{3}$, and TIMP1 and the fold change decrease in PDGF-BB were beyond \pm 1.5 (plotted in Fig. 4b; data shown in supplemental Tables S2, S3 and Supplemental Fig.S2). KEGG pathway analysis predicted that multiple pathways were significantly related to this altered cytokine profiling after SLC7A5 knockdown (supplemental Table S4). Among them, it is of particular interest that these pathways also included inflammatory bowel disease (IBD) and rheumatoid arthritis (Fig. 4c).

\section{Upregulated SLC7A5 activates mTOR-P70S6K signaling and enhances MMP3 and MMP13 expression in FLS}

To reveal the mechanism underlying MMP3 and MMP13 regulation at the protein level by SLC7A5, we detected amino acid sensor mTOR and its substrate in synovial tissues and inflamed FLSs. As shown in Fig. 5a, the expression of P70S6K and p-mTOR was significantly upregulated in RA synovial tissues compared with that of OA synovial tissues. IL- $1 \beta$ treatment led to the increased expression of SLC7A5 in FLS accompanied by P70S6K and 4EBP1 phosphorylation (Fig. 5b, Supplementary Fig. S5A). To confirm these results associated with the SLC7A5 role in amino acid sensor activation and signaling pathways involved in the regulation of translation, SLC7A5 was knocked down by RNAi in FLS. We found that phosphorylation of mTOR, P70S6K, and 4EBP1 was significantly intervened by si-SLC7A5 in IL$1 \beta$ treatment groups (Fig. 5c, Supplementary Fig. S5B). By using mTOR complex 1 (mTORC1) signal inhibitor rapamycin, protein levels of both MMP3 and MMP13 were also decreased significantly (Fig. 5d, Supplementary Fig. S5C). These findings suggest that the overexpressed SLC7A5 in FLS from RA patients has a crucial role in 


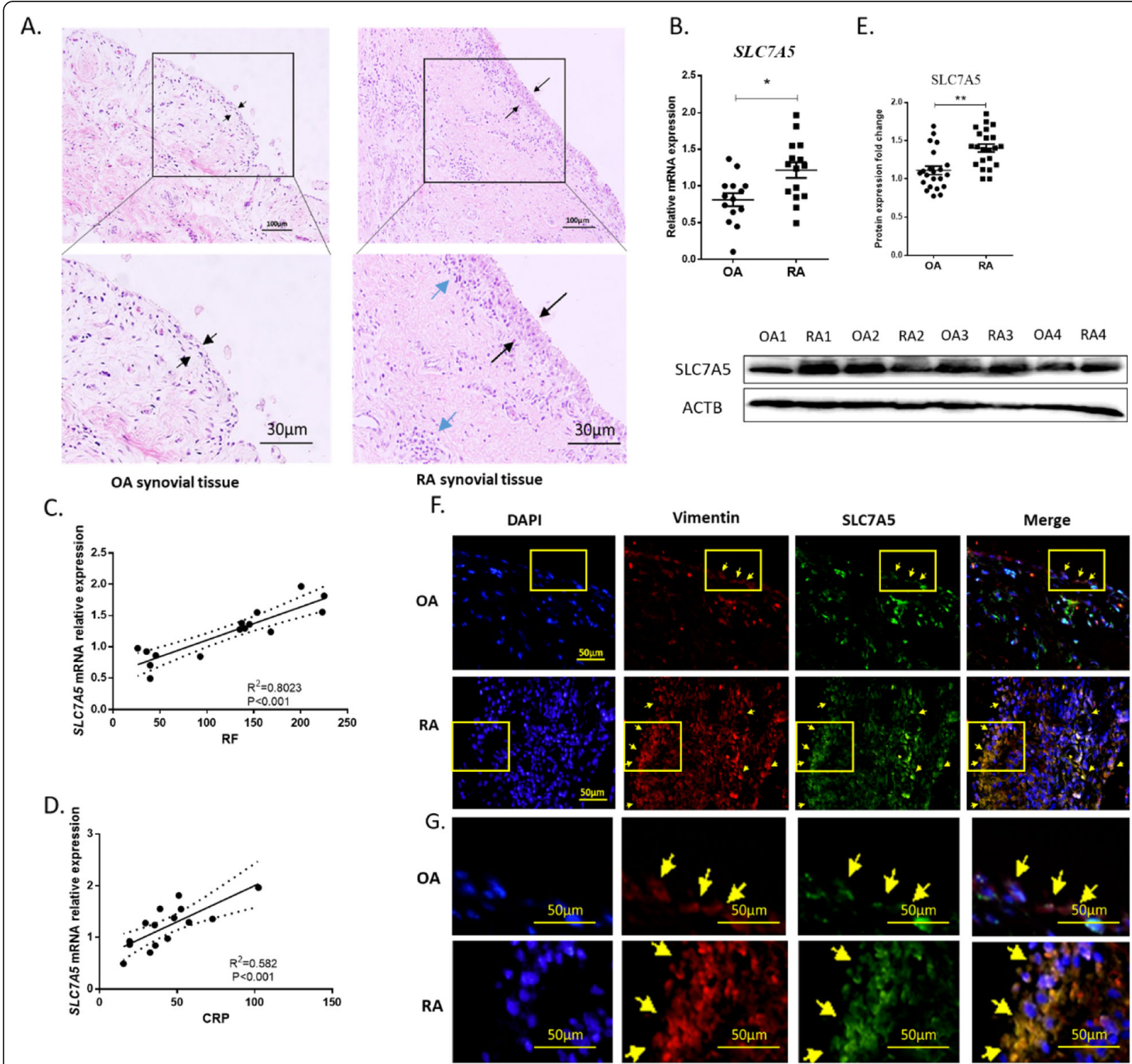

Fig. 1 SLC7A5 expression in fibroblast-like synoviocytes of RA patients. a Hematoxylin and eosin (H\&E) staining of the synovial tissue from RA and OA patients. Black arrows represent the lining cells of synovial tissues, the blue arrows represent the inflamed cells in synovial tissues. $\mathbf{b}$ The mRNA expression of SLC7A5 in synovial tissue from OA and RA patients, detected by RT-qPCR (RA $n=15, \mathrm{OA} n=14)$. Correlation analysis of SLC7A5 mRNA expression in FLS from RA patients $(n=15)$ with RF (c) and CRP (d). e The protein expression of SLC7A5 in synovial tissues from OA and RA patients detected by Western blotting. The density of SLC7A5 immune-reactive bands was analyzed by using ACTB expression as a loading control (RA $n=22, O A n=22$ ). f Representative immunofluorescence staining for SLC7A5 (green) and vimentin (red) in synovial tissue from OA and RA patients (RA $n=3, O A n=3$ ). The slide used for IF stain was consecutively followed slide stained with H\&E. The picture shown in $\mathbf{f}$ was the enlarging arrow area pointed out in $\mathbf{a}$. The yellow boxes were amplified in $\mathbf{g}$, yellow arrow pointed out the representative staining cells $\left({ }^{*} p<0.05\right)$

the activation of the mTORC1 pathway and subsequent regulation of the mRNA translation.

\section{Tryptophan stimulates mTOR activity and enhances} MMP3 and MMP13 expression in RA FLS

Downregulation of SLC7A5 (either by siRNA or through antibody blocking) led to the decreased expression of
MMP3 and MMP13 via inhibition of mTORC1 signaling in IL-1 $\beta$ treated FLS. We speculated the possible role of the amino acid transported via SLC7A5 in regulating MMP3 and MMP13 expression via mTORC1 signaling. To check that, extra amino acids (Phe, Trp, or Trp metabolic product Kyn) were added into the culture medium. As shown in Fig. 6a-f, only MMP13 could be 


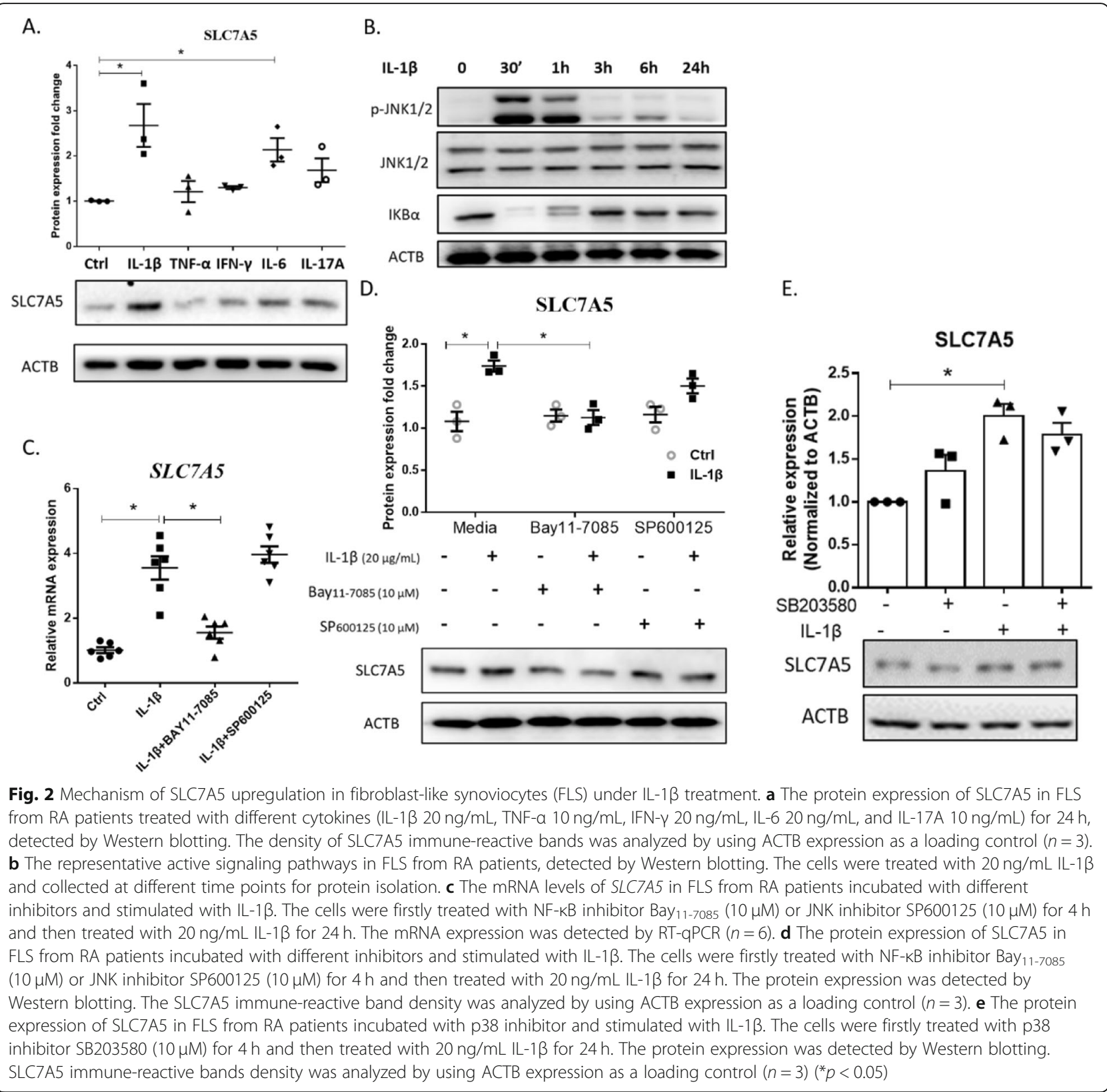

upregulated significantly in the IL- $1 \beta$ treatment group after the addition of extra Trp or Kyn (Supplementary Fig. S6). Meanwhile, the addition of extra Trp or Kyn could active the mTOR complex 1 signaling, as measured by 4EBP1 phosphorylation (Fig. 6d). For further investigations, FLSs were cultured in single amino acid (Phe or Trp)-deficient medium. We observed that the expression levels of both MMP3 and MMP13 were decreased significantly in the IL-1 $\beta$ treatment groups, under Trp deficiency (Fig. 6k, l), along with the reduction of the phosphorylated mTOR, P70S6K, and 4EBP1 (Fig. $6 \mathrm{~h}-\mathrm{j}$ ). All these data suggest that the upregulated
SLC7A5 may transport more special amino acid like Trp and regulate the MMP3 and MMP13 protein expression.

\section{Discussion}

In the present study, we demonstrated that the highly expressed SLC7A5 in the synoviocytes is induced by proinflammatory IL-1 $\beta$ via NF- $\mathrm{BB}$ signaling activation. Overexpressed SLC7A5 promotes mTOR-P70S6K signals and enhances the expression of MMP3 and MMP13 at the protein level in RA FLS.

Studies regarding the extent of metabolic changes and the types of metabolites involved may provide us suitable 


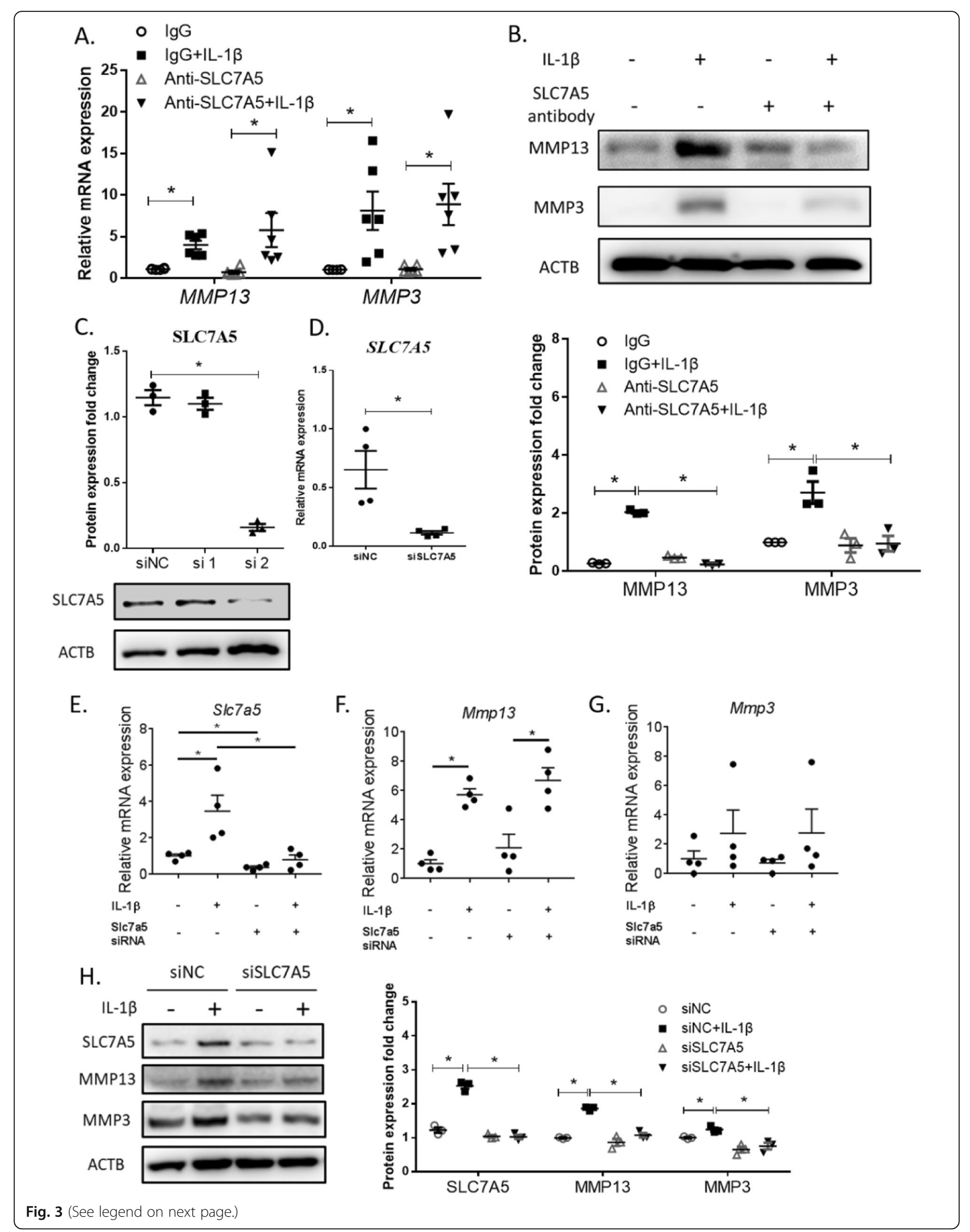




\begin{abstract}
(See figure on previous page.)
Fig. 3 Impact of SLC7A5 intervention either by antibody blocking or siRNA knockdown on the expression levels of MMP3 and MMP13 in FLS from RA patients. a The mRNA expression of MMP13 and MMP3 in RA FLS, incubated with SLC7A5 monoclonal antibody or isotype IgG and stimulated with or without IL-1 $\beta$. The cells were incubated with SLC7A5 monoclonal antibody or isotype lgG for $4 \mathrm{~h}$ and then treated with or without IL-1 $\beta(20 \mathrm{ng} / \mathrm{mL})$ for $8 \mathrm{~h}$. The mRNA levels were measured by RT-qPCR $(n=6)$. b The protein expression of MMP13 and MMP3 in RA FLS, incubated with SLC7A5 monoclonal antibody or isotype lgG and stimulated with or without IL-1 $\beta$. The cells were incubated with SLC7A5 monoclonal antibody or isotype lgG $4 \mathrm{~h}$ and then treated with or without IL-1 $\beta(20 \mathrm{ng} / \mathrm{mL})$ for $24 \mathrm{~h}$. The protein levels were detected by Western blotting. The density of MMP13 and MMP3 immune-reactive bands was analyzed by using ACTB expression as a loading control $(n=3)$. $\mathbf{c}, \mathbf{d}$ Optimization of the SLC7A5 RNAi efficiency in FLS from RA patients. The cells were transfected with siRNA (NC, siSLC7A5-1 or 2) for 24 h, and the protein and mRNA expression levels were detected by Western blotting (c) and RT-qPCR (d), respectively. e-g The expression of SLC7A5, MMP13, and MMP3 in FLS from RA patients transfected with siRNA (NC or siSLC7A5-2) and treated with or without IL-1 $\beta$, detected at the mRNA level by RT-qPCR. The cells were first transfected with siRNA (NC or siSLC7A5-2) for $24 \mathrm{~h}$ and then treated with or without IL-1 3 (20 $\mathrm{ng} / \mathrm{mL})$ for $8 \mathrm{~h}$ without changing the culture medium. $\mathbf{h}$ The protein expression of SLC7A5, MMP13, and MMP3 in FLS from RA patients transfected with siRNA (NC or siSLC7A5-2) and treated with or without IL-1 $\beta$. The cells were first transfected with siRNA (NC or siSLC7A5-2) for $24 \mathrm{~h}$ and then treated with or without IL-1 $\beta(20 \mathrm{ng} / \mathrm{mL})$ for $24 \mathrm{~h}$ further, without changing the culture medium. The density of MMP13 and MMP3 immune-reactive bands was analyzed by using ACTB expression as a loading control $(n=3)\left({ }^{*} p<0.05\right)$
\end{abstract}

biomarkers for RA diagnosis. Mounting evidence supports the notion that the metabolic changes occurring in the pathogenesis of RA are different from those found in other arthritis diseases [17]. Multiple amino acids such as glycine, leucine, serine, tyrosine, isoleucine, and proline have been reported in the synovial fluid of patients with RA [18]. However, only a few studies are available focusing on the involvement of amino acid transporter in RA pathogenesis. The metabolic changes in joint inflammation are complicated, and many interactions may take place, leading to a complex communication network between different cell types. Therefore, more knowledge is needed to unveil the critical interactions between amino acid transporter and FLS function in the arthritis process. In this study, we established that an amino acid transporter, SLC7A5, is overexpressed in FLS of RA patients, resulting in the upregulation of the MMPs at the protein level, which plays a critical role in maintaining FLS invasive phenotype and degradation of the extracellular matrix. Blocking SLC7A5 activity may slow down the FLS infiltration hence reducing MMP3 and MMP13 expression in RA development.

To understand the mechanism of SLC7A5 upregulation in RA FLS, the FLS inflammatory microenvironment was studied in this study. In RA synovial fluid, a lot of inflammatory mediators are secreted by immune cells. IFN- $\gamma$ and TNF- $\alpha$ are secreted by activated T cells [19]. IL-17 is produced by Th17 and mast cells [20]. Activated macrophages have been reported to secrete other cytokines like IL-1 $\beta$, IL-6, and TNF- $\alpha$ [21]. These inflammatory cytokines are well known to accelerate the process of matrix degradation in RA. Hence, we used a series of cytokines to stimulate FLS and observed the behavior of SLC7A5 in vitro. We found that IL-1 $\beta$-treated cells exhibited upregulated SLC7A5 expression via NF$\kappa B$ activation. Yoon et al. have recently demonstrated that SLC7A5 expression was upregulated by LPS in RA monocytes [22]. More interestingly, HIF-2 $\alpha$ was found binding to the Slc7a5 promoter and increased the Slc7a5 expression in normal liver and lung tissues [12]. Hypoxia is an important micro-environmental characteristic of RA, and hypoxia-inducible factors (HIFs) are key transcriptional factors that are highly expressed in RA synovium and are reported to modulate the expression of mediators that are involved in cellular infiltration of the synovial tissue, cartilage destruction, and bone erosion [23]. These findings suggested the potential role of SLC7A5 in RA pathogenesis.

To investigate the function of overexpressed SLC7A5 in RA, we focused on the amino acid transportability of this molecule. We hypothesized that amino acid transport may modulate the FLS infiltration in RA. Our data shows that siRNA knockdown0 or antibody blocking of SLC7A5 suppressed the protein levels of MMP3 and MMP13 significantly. And these two proteinases are responsible to mediate the cleavage of aggrecan and collagen in damaged cartilage [24]. Raposo et al. found that using the amino acid transport inhibitor 2-(methylamino) isobutyric acid could attenuate the severity of arthritis in diseased animals [25], suggesting that the amino acids and their transporters might be the key factor in RA pathogenesis. As we mentioned before, some studies suggest the synovial infiltration and tumor celllike behavior of FLS in RA. Likewise, many SLC7A5related tumor studies have demonstrated a critical role of SLC7A5 in tumor migration and invasion. For example, Janpipatkul et al. showed that the downregulation of the SLC7A5 expression suppressed cholangiocarcinoma cell migration and invasion [26]. Further, SLC7A5 regulated by miR-126-3p exhibited a strong association with cellular migration and metastasis in thyroid cancer cells [27]. All these data support the participation of SLC7A5 in regulating cellular infiltration and invasion, and MMP expression in RA FLS. We observed that antibody blocking of SLC7A5 suppressed the MMP3 and MMP13 expression only at the protein level, and not affected their mRNA levels, suggesting that blocked SLC7A5 might contribute to modulate the amino acid- 


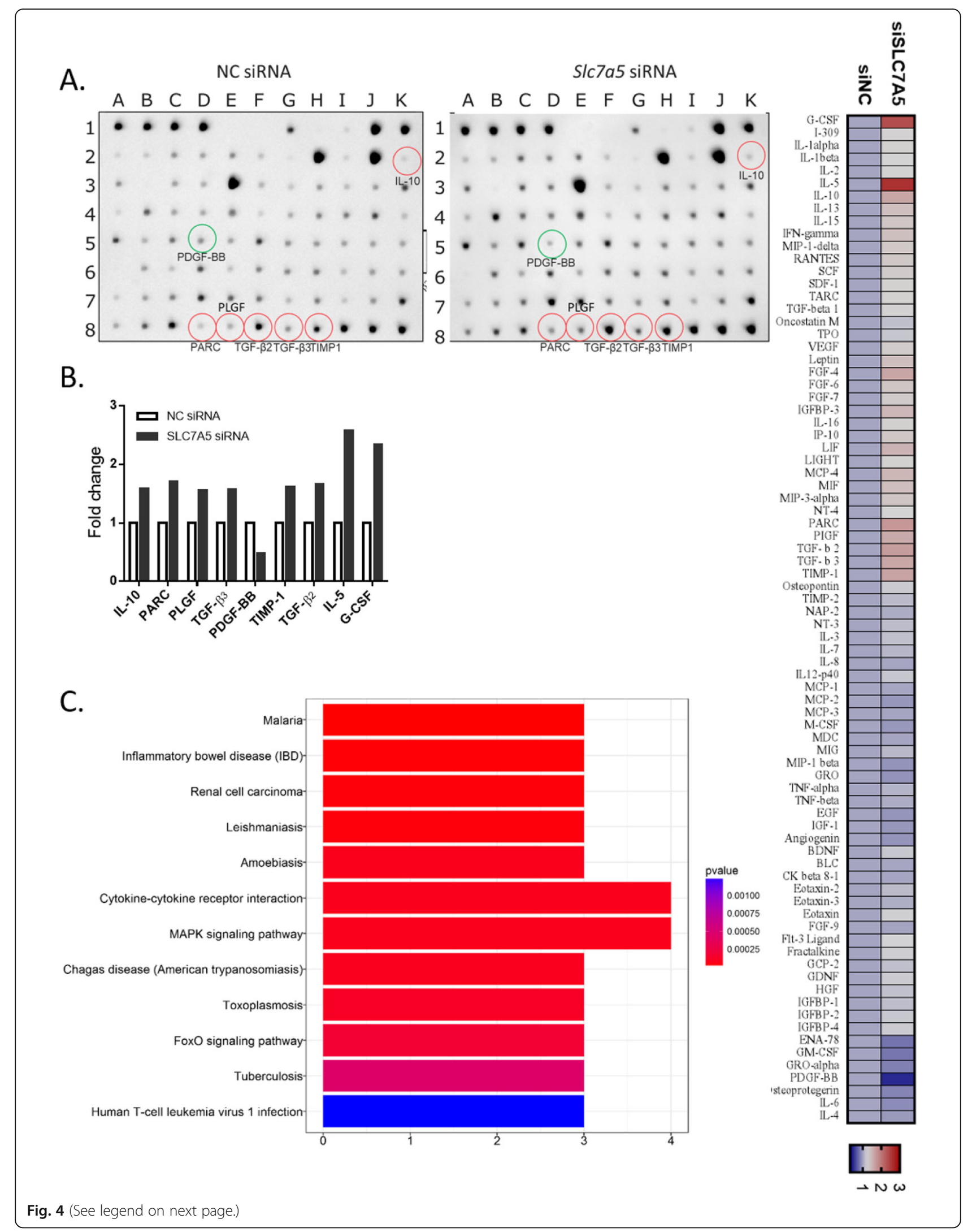


(See figure on previous page.)

Fig. 4 SLC7A5 impact on the production of cytokines and chemokines in RA FLS. a Images of cytokine array membranes incubated with supernatants from RA FLS, transfected with NC or SLC7A5 siRNA (si-2). The cells were transfected with either negative control or SLC7A5 siRNA (si-2) for $48 \mathrm{~h}$. All the cytokine and chemokine fold change is shown in the heat map. b Semi-quantitative data showing altered cytokine expression (fold change beyond \pm 1.5 ) in RA FLS supernatants $48 \mathrm{~h}$ post-siRNA transfection. $\mathbf{c}$ KEGG pathway analysis of the differentially expressed cytokines and chemokines in RA FLS

sensing mechanism. mTOR is a central nutrient sensor that signals a cell to grow and proliferate. One of the important functions of the mTOR complex (mTORC) is to maintain the available amino acid pool by regulating protein translation [28]. Dysregulation of the mTOR pathway leads to aberrant protein translation which manifests into various pathological states [29]. We showed that knocked down by siRNA, SLC7A5 could decrease the protein level of MMP3 and MMP13 via suppressing the phosphorylation of mTOR and P70S6K significantly. MMP3 and MMP13 expression was also decreased significantly when we used rapamycin to inhibit mTORC1 signaling. Ito et al. have shown that knockdown of Raptor (a component of mTORC1), reduced the MMP3 and MMP13 expression in nucleus pulposus cells of the human intervertebral disc treated with IL-1 $\beta$ [30]. Cejka et al. showed that inhibited mTORC1 via sirolimus or everolimus could reduce synovial osteoclast formation and protect against local bone erosions and cartilage loss [31]. Curcumin, another inhibitor of mTOR signaling, was also reported to alleviate rheumatoid arthritis-induced inflammation and synovial hyperplasia by reducing inflammatory mediators like IL$1 \beta$, TNF- $\alpha$, MMP-1, and MMP-3 [32]. SLC7A5 mediates amino acid flux and activates mTORC1 signaling in tumors as well as immune cells [12, 22]. SLC7A5 knockout

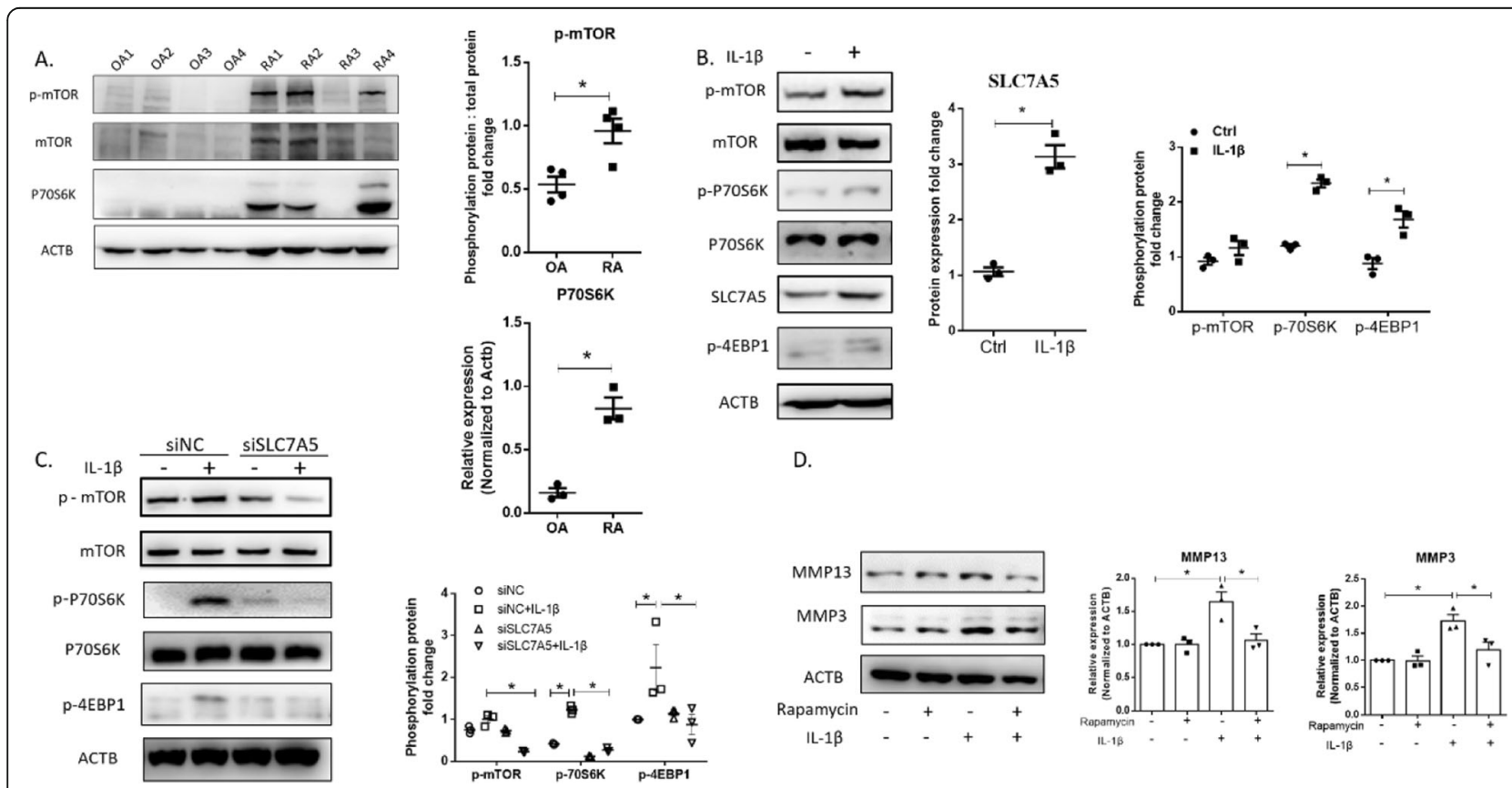

Fig. 5 Activation of mTOR-P70S6K signaling and downstream upregulation of MMP3 and MMP13 expression by SLC7A5 overexpressed in RA FLS. a The protein expression of p-mTOR $(n=4)$, mTOR, and P70S6K $(n=3)$ in synovial tissue from OA and RA patients detected by Western blotting. b The protein synthesis pathway activation in FLS. The FLS were treated with $20 \mathrm{ng} / \mathrm{mL} \mathrm{IL}-1 \beta$ for $4 \mathrm{~h}$ and collected for protein isolation, detected by Western blotting. The density of SLC7A5 immune-reactive bands was analyzed by using ACTB expression as a loading control. The phosphorylation protein ratio fold change of mTOR and P70S6K was analyzed by using total protein expression of their own as a control, while the phosphorylation protein ratio fold change of 4EBP1 was analyzed by using ACTB expression as a loading control $(n=3)$. c The impact of SLC7A5 siRNA on the protein synthesis pathway (mTOR-P70S6K-4EBP1) activation in FLS, detected by Western blotting. The cells were transfected with siNC or siSLC7A5 (si-2) for $24 \mathrm{~h}$ and then stimulated with $20 \mathrm{ng} / \mathrm{mL} \mathrm{IL}-1 \beta$ for another $4 \mathrm{~h}$. The fold change in phosphorylated/nonphosphorylated protein ratios of mTOR and P70S6K was analyzed by using total protein expression of their own as a control, while that of 4EBP1 was analyzed by using ACTB expression as a loading control $(n=3)$. $\mathbf{d}$ The inhibition of MMP3 and MMP13 expression by rapamycin (mTORC1 inhibitor) in RA FLS under IL-1 $\beta$ treatment. The cells were incubated with rapamycin (100 nM) for $8 \mathrm{~h}$ and then stimulated with $20 \mathrm{ng} / \mathrm{mL} \mathrm{IL}-1 \beta \mathrm{for}$ another $24 \mathrm{~h}$. The protein levels were detected by Western blotting. The density of MMP3 and MMP13 immune-reactive bands was analyzed by using ACTB expression as a loading control $(n=3)\left({ }^{*} p<0.05\right)$ 


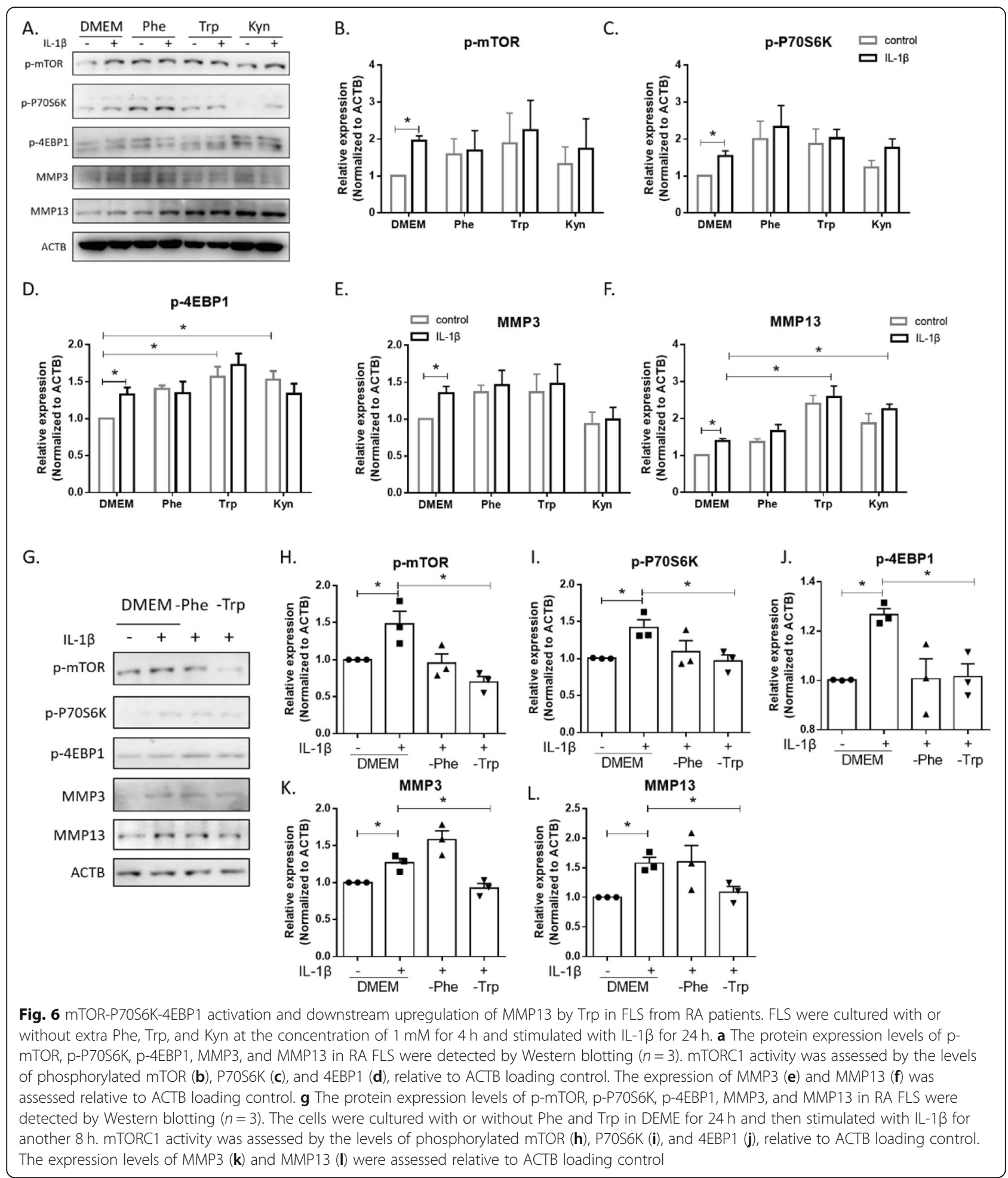

cancer cell lines showed decreased P70S6K phosphorylation and compromised cell proliferation [33]. Intracellular amino acids induce mTOR phosphorylation which activates its downstream target P70S6K [34]. P70S6K plays important roles in cell growth, proliferation, and differentiation by regulating cell cycle progression and ribosome biogenesis $[35,36]$. It phosphorylates multiple components of the translational machinery and related regulators and increases translation by stimulating rRNA and tRNA synthesis [37]. It is reported that SLC7A5 
together with SLC3A2 participates in transporting large neutral amino acids such as Phe, tyrosine, or Trp into the cell [38]. As shown in the present study, FLS cultured in a Trp-deficient medium exhibited a decreased expression of MMP13. In contrast, when FLS were cultured in the Trp supplemented medium, the expression of MMP13 was increased. At the same time, the supplemented Trp could activate the mTOR complex 1 signaling. Likewise, Kyn (a metabolite of Trp) was also able to activate mTOR signaling in T cells of SLE patients [39], suggesting that amino acids and their metabolites may also play important roles in regulating autoimmune response. Moreover, the upstream of mTOR signal, Akt activation, could also induce MMP3 and MMP13 expression in the microglia [40]. In the present study, we also found that SLC7A5 siRNA could cause increased IL-10 and TIMP1 and decreased PDGF-BB protein production in RA FLS supernatant. Previously, it was reported that IL-10 works as an anti-inflammatory cytokine, inhibits VEGF [41], and suppresses inflammatory response [42]. The increased Timp1 has been reported to ameliorate cartilage destruction in collageninduced arthritis in rats [43]. All these findings are in accordance with our hypothesis that the amino acid transporter SLC7A5 takes part in cellular invasion and regulates protein levels of MMP3 and MMP13 via the mTOR signaling pathway in RA FLS. Downregulated or blocked SLC7A5 in FLS could serve as an antiinflammatory molecule and a potential therapeutic target in arthritis.

\section{Conclusion}

The present study highlights the important function of SLC7A5 in FLS from RA patients. IL-1 $\beta$ treatment of the cells causes higher expression of SLC7A5 through the NF- $\mathrm{kB}$ pathway. Blocking SLC7A5 activity inhibits MMP3 and MMP13 expression in FLS. Contrarily, overexpressed SLC7A5 enhances the protein production of MMP and MMP13 mediated by the mTOR-P70S6Ktranslation pathway. The findings provide new insights into the pathogenesis of RA and may pave the way for novel therapeutic strategies for the treatment of the disease.

\section{Supplementary information}

Supplementary information accompanies this paper at https://doi.org/10. 1186/s13075-020-02296-8.

Additional file 1 : Fig. S1. The SLC7A5 expression in RA and OA synovial tissues. Fig. S2. The differentially expressed cytokines and chemokines affected by SLC7A5 in RA FLS were analyzed by GO terms. (A). biological process, (B). molecular function, (C). cellular component. Fig. S3. The protein expression of SLC7A5 in FLS from RA patients incubated with different inhibitors and stimulated with IL-1 $\beta$. Fig. S4. The protein expression of MMP3, MMP13 and SLC7A5 in FLS from RA patients blocked via SLC7A5 antibody (A) or siRNA (B). Fig. S5. Activation of mTOR-P70S6K signalling and downstream up-regulation of MMP3 and MMP13 expression by SLC7A5 over expressed in RA FLS. The mTOR-

P70S6K signal activation after IL-1 $\beta$ treatment (A). The impact of SLC7A5 siRNA on the protein synthesis pathway (mTOR-P70S6K-4EBP1) activation in FLS (B). The inhibition of MMP3 and MMP13 expression by rapamycin (mTORC1 inhibitor) in RA FLS under IL-1 $\beta$ treatment (C). Fig. S6. mTORP70S6K-4EBP1 activation and downstream up-regulation of MMP13 by Trp in FLS from RA patients. Table S1. Primer. Table S2. Cytokine dot ELISA list. Table S3. Cytokine expression. Table S4. KEGG pathway analysis. Table S5. Primary antibodies.

\section{Abbreviations}

CRP: C-reactive protein; ESR: Erythrocyte sedimentation rate; FLS: Fibroblastlike synoviocytes; Kyn: Kynurenine; MMPs: Matrix metalloproteinases; mTORC1: Mechanistic target of rapamycin complex 1; Phe: Phenylalanine; RA: Rheumatoid arthritis; RF: Rheumatoid factor; SLC7A5: Solute carrier family 7 member 5; Trp: Tryptophan

\section{Acknowledgements \\ We are grateful to Dr. Safdar Hussain for the careful proofreading of our manuscript.}

\section{Authors' contributions}

Jing $\mathrm{Xu}$, Congshan Jiang, and Shemin Lu conceived, designed, and executed the research; analyzed the data, and wrote the paper. Yongsong Cai, Jiawen $\mathrm{Xu}, \mathrm{Ke} \mathrm{Xu}$, and Peng Xu provided the synovial tissues and persuaded patients to donate their tissue samples and share their medical information. Jing Xu, Congshan Jiang, Yuanxu Guo, Xipeng Wang, Jiaxiang Zhang, Wenhua Zhu, Si Wang, Fujun Zhang, Manman Geng, Yan Han, Qilan Ning, and Liesu Meng contributed to the acquisition and/or analysis of the data. All the authors approved the final version of the paper.

\section{Funding}

This work was supported by grants from the National Natural Science Foundation of China (No. 81671629 and No. 81701619) and the Fundamental Research Funds for the Central Universities (xjj2017143 and syspz2017006). No financial support or other benefits from commercial sources were obtained for this work.

\section{Availability of data and materials}

The data sets used and/or analyzed during the current study are available from the corresponding author on reasonable request.

\section{Ethics approval and consent to participate}

Written informed consent was obtained from all the patients. This study was approved by the Medical Ethics Committee of Xi'an Jiaotong University (No. 2016-261 and No.2017-666).

\section{Consent for publication}

Not applicable.

\section{Competing interests}

Authors declare that they have no competing financial interests.

\section{Author details}

${ }^{1}$ Department of Biochemistry and Molecular Biology, School of Basic Medical Sciences, Xi'an Jiaotong University Health Science Center, Xi'an 710061, Shaanxi, People's Republic of China. ${ }^{2}$ Key Laboratory of Environment and Genes Related to Diseases (Xi'an Jiaotong University), Ministry of Education, Xi'an 710061, Shaanxi, People's Republic of China. ${ }^{3}$ Department of Joint Surgery, Xi'an Hong Hui Hospital, Xi'an Jiaotong University Health Science Center, Xi'an 710061, Shaanxi, People's Republic of China.

Received: 31 March 2020 Accepted: 19 August 2020

Published online: 31 August 2020

References

1. Vos T, Flaxman AD, Naghavi M, Lozano R, Michaud C, Ezzati M, Shibuya K, Salomon JA, Abdalla S, Aboyans V, et al. Years lived with disability (YLDs) for 
1160 sequelae of 289 diseases and injuries 1990-2010: a systematic analysis for the Global Burden of Disease Study 2010. Lancet. 2012;380(9859):216396.

2. Bartok B, Firestein GS. Fibroblast-like synoviocytes: key effector cells in rheumatoid arthritis. Immunol Rev. 2010;233(1):233-55.

3. Fassbender HG, Simmling-Annefeld $M$. The potential aggressiveness of synovial tissue in rheumatoid arthritis. J Pathol. 1983;139(3):399-406.

4. Li X, Makarov SS. An essential role of NF-KB in the "tumor-like" phenotype of arthritic synoviocytes. Proc Natl Acad Sci. 2006;103(46):17432-7.

5. Zeisel MB, Druet VA, Wachsmann D, Sibilia J. MMP-3 expression and release by rheumatoid arthritis fibroblast-like synoviocytes induced with a bacterial ligand of integrin a5ß1. Arthritis Res Ther. 2004;7(1):R118.

6. Galluccio M, Pingitore $\mathrm{P}$, Scalise M, Indiveri C. Cloning, large scale overexpression in E. coli and purification of the components of the human LAT 1 (SLC7A5) amino acid transporter. Protein J. 2013;32(6):442-8.

7. Mastroberardino L, Spindler B, Pfeiffer R, Skelly PJ, Loffing J, Shoemaker CB, Verrey F. Amino-acid transport by heterodimers of 4F2hc/CD98 and members of a permease family. Nature. 1998;395(6699):288-91.

8. Yanagida O, Kanai Y, Chairoungdua A, Kim DK, Segawa H, Nii T, Cha SH, Matsuo H, Fukushima J, Fukasawa Y, et al. Human L-type amino acid transporter 1 (LAT1): characterization of function and expression in tumor cell lines. Biochim Biophys Acta. 2001;1514(2):291-302.

9. Poncet N, Mitchell FE, Ibrahim AF, McGuire VA, English G, Arthur JS, Shi YB, Taylor PM. The catalytic subunit of the system L1 amino acid transporter (slc7a5) facilitates nutrient signalling in mouse skeletal muscle. PLoS One. 2014;9(2):e89547.

10. Sato Y, Heimeier RA, Li C, Deng C, Shi YB. Extracellular domain of CD98hc is required for early murine development. Cell Biosci. 2011;1(1):7.

11. Sinclair LV, Rolf J, Emslie E, Shi YB, Taylor PM, Cantrell DA. Control of amino-acid transport by antigen receptors coordinates the metabolic reprogramming essential for T cell differentiation. Nat Immunol. 2013;14(5):500-8.

12. Elorza A, Soro-Arnaiz I, Melendez-Rodriguez F, Rodriguez-Vaello V, Marsboom G, de Carcer G, Acosta-lborra B, Albacete-Albacete L, Ordonez A, Serrano-Oviedo L, et al. HIF2alpha acts as an mTORC1 activator through the amino acid carrier SLC7A5. Mol Cell. 2012;48(5):681-91.

13. Brenchley PE. Angiogenesis in inflammatory joint disease: a target for therapeutic intervention. Clin Exp Immunol. 2000;121(3):426-9.

14. Yu Z, Lin W, Rui Z, Jihong P. Fibroblast-like synoviocyte migration is enhanced by IL17-mediated overexpression of L-type amino acid transporter 1 (LAT1) via the mTOR/4E-BP1 pathway. Amino Acids. 2018:50(2):331-40.

15. Lin L, Taktakishvili O, Talman W. Colocalization of neurokinin-1, N-methyl-Daspartate, and AMPA receptors on neurons of the rat nucleus tractus solitarii. Neuroscience. 2008;154(2):690-700.

16. Zhu W, Jiang C, Xu J, Geng M, Wu X, Sun J, Ma J, Holmdahl R, Meng L, Lu S. Pristane primed rat T cells enhance TLR3 expression of fibroblast-like synoviocytes via TNF-a initiated p38 MAPK and NF-kB pathways. Clin Immunol. 2015;156(2):141-53.

17. Chimenti MS, Triggianese P, Conigliaro P, Candi E, Melino G, Perricone R. The interplay between inflammation and metabolism in rheumatoid arthritis. Cell Death Dis. 2015;6:e1887.

18. Priori R, Scrivo R, Brandt J, Valerio M, Casadei L, Valesini G, Manetti C. Metabolomics in rheumatic diseases: the potential of an emerging methodology for improved patient diagnosis, prognosis, and treatment efficacy. Autoimmun Rev. 2013;12(10):1022-30.

19. Su Z, Yang R, Zhang W, Xu L, Zhong Y, Yin Y, Cen J, DeWitt JP, Wei Q. The synergistic interaction between the calcineurin B subunit and IFN-gamma enhances macrophage antitumor activity. Cell Death Dis. 2015;6:e1740.

20. Kotake S, Udagawa N, Takahashi N, Matsuzaki K, Itoh K, Ishiyama S, Saito S, Inoue K, Kamatani N, Gillespie MT, et al. IL-17 in synovial fluids from patients with rheumatoid arthritis is a potent stimulator of osteoclastogenesis. J Clin Invest. 1999;103(9):1345-52.

21. Zhu W, Xu J, Jiang C, Wang B, Geng M, Wu X, Hussain N, Gao N, Han Y, Li $D$, et al. Pristane induces autophagy in macrophages, promoting a STAT1IRF1-TLR3 pathway and arthritis. Clin Immunol. 2017;175:56-68.

22. Yoon BR, Oh YJ, Kang SW, Lee EB, Lee WW. Role of SLC7A5 in metabolic reprogramming of human monocyte/macrophage immune responses. Front Immunol. 2018;9:53.

23. Hua S, Dias TH. Hypoxia-inducible factor (HIF) as a target for novel therapies in rheumatoid arthritis. Front Pharmacol. 2016;7:184.

24. Troeberg L, Nagase $H$. Proteases involved in cartilage matrix degradation in osteoarthritis. Biochim Biophys Acta. 2012;1824(1):133-45.
25. Raposo B, Vaartjes D, Ahlqvist E, Nandakumar KS, Holmdahl R. System A amino acid transporters regulate glutamine uptake and attenuate antibodymediated arthritis. Immunology. 2015;146(4):607-17.

26. Janpipatkul K, Suksen K, Borwornpinyo S, Jearawiriyapaisarn N, Hongeng S, Piyachaturawat $P$, Chairoungdua A. Downregulation of LAT1 expression suppresses cholangiocarcinoma cell invasion and migration. Cell Signal. 2014;26(8):1668-79.

27. Xiong Y, Kotian S, Zeiger MA, Zhang L, Kebebew E. miR-126-3p inhibits thyroid cancer cell growth and metastasis, and is associated with aggressive thyroid cancer. PLoS One. 2015;10(8):e0130496.

28. Hara K, Yonezawa K, Weng QP, Kozlowski MT, Belham C, Avruch J. Amino acid sufficiency and mTOR regulate p70 S6 kinase and elF-4E BP1 through a common effector mechanism. J Biol Chem. 1998;273(23):14484-94.

29. Laplante M, Sabatini DM. mTOR signaling in growth control and disease. Cell. 2012;149(2):274-93.

30. Ito M, Yurube T, Kakutani K, Maeno K, Takada T, Terashima Y, Kakiuchi Y, Takeoka Y, Miyazaki S, Kuroda R, et al. Selective interference of mTORC1/ RAPTOR protects against human disc cellular apoptosis, senescence, and extracellular matrix catabolism with Akt and autophagy induction. Osteoarthr Cartil. 2017;25(12):2134-46.

31. Cejka D, Hayer S, Niederreiter B, Sieghart W, Fuereder T, Zwerina J, Schett G. Mammalian target of rapamycin signaling is crucial for joint destruction in experimental arthritis and is activated in osteoclasts from patients with rheumatoid arthritis. Arthritis Rheum. 2010;62(8):2294-302.

32. Dai Q, Zhou D, Xu L, Song X. Curcumin alleviates rheumatoid arthritisinduced inflammation and synovial hyperplasia by targeting mTOR pathway in rats. Drug Design Dev Ther. 2018;12:4095-105.

33. Cormerais Y, Giuliano S, LeFloch R, Front B, Durivault J, Tambutte E, Massard PA, de la Ballina LR, Endou $H$, Wempe MF, et al. Genetic disruption of the multifunctional CD98/LAT1 complex demonstrates the key role of essential amino acid transport in the control of mTORC1 and tumor growth. Cancer Res. 2016;76(15):4481-92.

34. Blommaart EF, Luiken JJ, Blommaart PJ, van Woerkom GM, Meijer AJ. Phosphorylation of ribosomal protein $\mathrm{S} 6$ is inhibitory for autophagy in isolated rat hepatocytes. J Biol Chem. 1995;270(5):2320-6.

35. Duvel K, Yecies JL, Menon S, Raman P, Lipovsky Al, Souza AL, Triantafellow $\mathrm{E}, \mathrm{Ma} \mathrm{Q}$, Gorski R, Cleaver $\mathrm{S}$, et al. Activation of a metabolic gene regulatory network downstream of mTOR complex 1. Mol Cell. 2010;39(2):171-83.

36. Shin S, Wolgamott L, Yu Y, Blenis J, Yoon SO. Glycogen synthase kinase (GSK)-3 promotes p70 ribosomal protein S6 kinase (p70S6K) activity and cell proliferation. Proc Natl Acad Sci U S A. 2011;108(47):E1204-13.

37. Morita M, Gravel SP, Hulea L, Larsson O, Pollak M, St-Pierre J, Topisirovic I. mTOR coordinates protein synthesis, mitochondrial activity and proliferation. Cell Cycle. 2015;14(4):473-80.

38. Friesema EC, Docter $R$, Moerings EP, Verrey F, Krenning EP, Hennemann G, Visser TJ. Thyroid hormone transport by the heterodimeric human system $\mathrm{L}$ amino acid transporter. Endocrinology. 2001;142(10):4339-48.

39. Perl A, Hanczko R, Lai ZW, Oaks Z, Kelly R, Borsuk R, Asara JM, Phillips PE. Comprehensive metabolome analyses reveal $\mathrm{N}$-acetylcysteine-responsive accumulation of kynurenine in systemic lupus erythematosus: implications for activation of the mechanistic target of rapamycin. Metabolomics. 2015; 11(5):1157-74

40. Ito S, Kimura K, Haneda M, Ishida $Y$, Sawada M, Isobe K. Induction of matrix metalloproteinases (MMP3, MMP12 and MMP13) expression in the microglia by amyloid-beta stimulation via the PI3K/Akt pathway. Exp Gerontol. 2007; 42(6):532-7.

41. Hong KH, Cho ML, Min SY, Shin YJ, Yoo SA, Choi JJ, Kim WU, Song SW, Cho CS. Effect of interleukin-4 on vascular endothelial growth factor production in rheumatoid synovial fibroblasts. Clin Exp Immunol. 2007;147(3):573-9.

42. Broeren MG, de Vries M, Bennink MB, Arntz OJ, van Lent PL, van der Kraan PM, van den Berg WB, van den Hoogen FH, Koenders MI, van de Loo FA. Suppression of the inflammatory response by disease-inducible interleukin10 gene therapy in a three-dimensional micromass model of the human synovial membrane. Arthritis Res Ther. 2016;18:186.

43. Guo J, Zhao W, Cao X, Yang H, Ding J, Ding J, Tan Z, Ma X, Hao C, Wu L, et al. SIRT1 promotes tumor-like invasion of fibroblast-like synoviocytes in rheumatoid arthritis via targeting TIMP1. Oncotarget. 2017:8(51):88965-73.

\section{Publisher's Note}

Springer Nature remains neutral with regard to jurisdictional claims in published maps and institutional affiliations. 